\title{
Quantitative Translational Analysis of Brain Kynurenic Acid Modulation via Irreversible Kynurenine Aminotransferase II Inhibition
}

\author{
Cheng Chang, ${ }^{1}$ Kari R. Fonseca, ${ }^{1}$ Cheryl Li, Weldon Horner, Laura E. Zawadzke, \\ Michelle A. Salafia, Kathryn A. Welch, Christine A. Strick, Brian M. Campbell, \\ Steve S. Gernhardt, Haojing Rong, Aarti Sawant-Basak, Jennifer Liras, Amy Dounay, \\ Jamison B. Tuttle, Patrick Verhoest, and Tristan S. Maurer
}

Systems Modeling and Simulation Group, Pharmacokinetics, Dynamics and Metabolism, Medicine Design (C.C., C.L., T.S.M.), Neuroscience and Pain Research Unit (W.H., L.E.Z., M.A.S., K.A.W., C.A.S., B.M.C., A.D., J.B.T., P.V.), and Pharmacokinetics, Dynamics and Metabolism, Medicine Design (K.R.F., S.S.G., H.R., A.S.-B., J.L.), Worldwide Research and Development, Pfizer Inc., Cambridge, Massachusetts

Received January 4, 2018; accepted May 24, 2018

\begin{abstract}
Kynurenic acid (KYNA) plays a significant role in maintaining normal brain function, and abnormalities in KYNA levels have been associated with various central nervous system disorders. Confirmation of its causality in human diseases requires safe and effective modulation of central KYNA levels in the clinic. The kynurenine aminotransferases (KAT) II enzyme represents an attractive target for pharmacologic modulation of central KYNA levels; however, KAT II and KYNA turnover kinetics, which could contribute to the duration of pharmacologic effect, have not been reported. In this study, the kinetics of central KYNA-lowering effect in rats and nonhuman primates (NHPs, Cynomolgus macaques) was investigated using multiple KAT II irreversible inhibitors as pharmacologic probes. Mechanistic pharmacokinetic-pharmacodynamic analysis of in vivo responses to irreversible inhibition quantitatively revealed that
\end{abstract}

1) KAT II turnover is relatively slow [16-76 hours' half-life $\left(t_{1 / 2}\right)$ ], whereas KYNA is cleared more rapidly from the brain $\left(<1\right.$ hour $\left.t_{1 / 2}\right)$ in both rats and NHPs, 2) KAT II turnover is slower in NHPs than in rats (76 hours vs. 16 hours $t_{1 / 2}$, respectively), and 3) the percent contribution of KAT II to KYNA formation is constant ( $80 \%)$ across rats and NHPs. Additionally, modeling results enabled establishment of in vitro-in vivo correlation for both enzyme turnover rates and drug potencies. In summary, quantitative translational analysis confirmed the feasibility of central KYNA modulation in humans. Model-based analysis, where system-specific properties and drug-specific properties are mechanistically separated from in vivo responses, enabled quantitative understanding of the KAT II-KYNA pathway, as well as assisted development of promising candidates to test KYNA hypothesis in humans.

\section{Introduction}

More than a century after kynurenic acid (KYNA) was first synthesized (Homer, 1914), extensive knowledge has accumulated about its biosynthesis and physiologic functions. KYNA was identified to be a competitive blocker of the glycine coagonist site of the NMDA ( $N$-methyl-daspartate) subtype of glutamate receptors (Kessler et al., 1989), as well as a noncompetitive antagonist of the $\alpha 7$-nicotinic acetylcholine receptor (Hilmas et al., 2001). Both receptors affect cognitive functions (Robbins and Murphy, 2006; Thomsen et al., 2010).

\footnotetext{
This work was supported by Pfizer Inc.

${ }^{1}$ C.C. and K.R.F. contributed equally to the manuscript.

https://doi.org/10.1124/mol.118.111625.
}

In fact, abnormally high levels of KYNA in both the brain and cerebrospinal fluid (CSF) have been associated with pathologic conditions such as schizophrenia, bipolar disease, and cognitive impairment (Erhardt et al., 2001; Schwarcz et al., 2001; Olsson et al., 2010). Whereas KYNA has been reported to be a weak antagonist at the glycine site of NMDA and $\alpha 7$, it has been shown to be a potent agonist at the aryl hydrocarbon receptor. The role of the aryl hydrocarbon receptor in the central nervous system and disease is somewhat unknown; however, activation of this receptor has proinflammatory effects with increases in interleukin-8, which have been linked to psychiatric disease (DiNatale et al., 2010). All evidence points to the significant role of KYNA in maintaining normal brain function. The ability to fine-tune brain KYNA levels is

ABBREVIATIONS: $\mathrm{Cl}$, confidence interval; CSF, cerebrospinal fluid; DMSO, dimethylsulfoxide; HBSS, Hanks' balanced salt solution; HPLC, highperformance liquid chromatography; IACUC, Institutional Animal Care and Use Committee; IVIVC, in vitro-in vivo correlation; KAT, kynurenine aminotransferase; KAT II, kynurenine aminotransferases II; KYNA, kynurenic acid; L-KYN, L-kynurenine; LC-MS/MS, liquid chromatography-tandem mass spectrometry; NHP, nonhuman primate; NMDA, N-methyl-daspartate; PD, pharmacodynamics; PK, pharmacokinetics; PKPD, pharmacokinetics pharmacodynamics; PLP, pyridoxal phosphate. 
critical to test the hypotheses of KYNA roles in different disease populations.

KYNA is one of the terminal metabolites of tryptophan, and its synthesis in the brain from L-kynurenine (L-KYN) is primarily by astrocytic kynurenine aminotransferases (KAT) II, with minor contributions from KAT I, KAT III, and KAT IV (Kiss et al., 2003; Han et al., 2010). KAT II has been considered the prime target for maintaining and regulating physiologic concentrations of brain KYNA (Guidetti et al., 1997, 2007; Amori et al., 2009). Irreversible KAT II inhibitors are effective modulators of brain KYNA levels (Dounay et al., 2012; Tuttle et al., 2012; Henderson et al., 2013). One such irreversible KAT II inhibitor (PF-04859989) has demonstrated procognitive effects in various preclinical models (Koshy Cherian et al., 2014; Kozak et al., 2014; Linderholm et al., 2016), suggesting a promising role in treating the cognitive symptoms associated with elevated KYNA levels.

Typically, irreversible inhibitors have been used as probes to understand enzyme mechanisms (Deigner et al., 1999; Rempel and Withers, 2008). In the current study, their use was expanded to quantify enzyme in vivo kinetic properties through mechanistic modeling. More specifically, in the process of characterizing KAT II irreversible inhibitors, new insight could emerge from quantitative analysis of the pharmacologically perturbed biologic system, which reveals the biology surrounding KAT II, as well as enables efficient drug design and development (Abelö et al., 2000). Quantitative evaluation of the in vivo responses has unique challenges, such as time-dependent target-site drug concentration, which requires a pharmacokinetic $(\mathrm{PK})$ model to characterize or the temporal disconnect between the inhibitor concentration and the subsequent pharmacodynamic (PD) effect, which requires a model that accounts for both the concentration and timedependent nature of the pharmacodynamics response (Abelö et al., 2000; Ferron et al., 2001) before in vivo potency can be reliably estimated. Mechanism-based PKPD analysis represents a powerful tool in this regard, where PK and PD are mathematically integrated to resolve drug-specific properties (e.g., drug distribution, binding potency) and biologic systemspecific properties (e.g., target turnover rate, signal transduction time) from in vivo responses (Danhof et al., 2007). Similar approaches have been successfully applied to understanding irreversible inhibition of gastric acid secretion by omeprazole (Abelo et al., 2000) and pantoprazole (Ferron et al., 2001). In the current study, four specific irreversible KAT II inhibitors (Fig. 1) were used as pharmacologic probes to study KAT II biologic pathway in both rats and nonhuman primates (NHPs). A mechanism-based PKPD model was applied to estimate the in vivo potencies of multiple irreversible inhibitors, as well as turn over the half-lives of KAT II and KYNA in rodents and NHPs, which has not previously been reported. Additionally, in vitro investigations of corresponding properties were carried out. The correlation between in vitro and in vivo estimations for both drug-specific and biologic system-specific properties is subsequently evaluated. Lastly, the contribution of KAT II-to-KYNA formation relative to other enzymes in the brain was also estimated in both species. Ultimately, a quantitative understanding of the underlying biology and molecular mechanism of action was exploited to identify compounds with the requisite properties to test the therapeutic potential of KATII inhibition in the clinic.

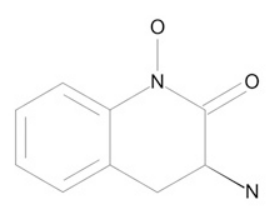

PF -04859989

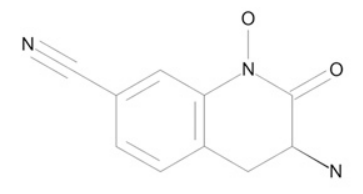

PF- 06253133

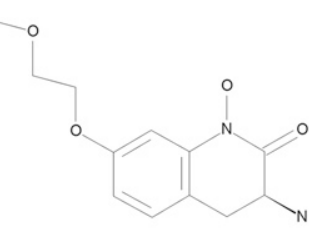

$P F-05579960$

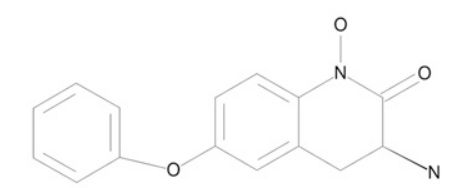

PF- 05182357
Fig. 1. The chemical structures of PF-04859989, PF-05579960, PF-06253133, and PF-05182357.

\section{Materials and Methods}

\section{Chemicals}

PF-04859989 ((3S)-3-amino-1-hydroxy-3,4-dihydroquinolin-2(1H)-one), PF-05579960 ((3S)-3-amino-1-hydroxy-7-(2-methoxyethoxy)-3,4-dihydroquinolin-2(1H)-one), PF-06253133 ((3S)-3-amino-1-hydroxy-2-oxo1,2,3,4-tetrahydroquinoline-7-carbonitrile), and PF-05182357 ((3S)-3-amino1-hydroxy-6-phenoxy-3,4-dihydroquinolin-2(1H)-one) were synthesized by the Pfizer Medicinal Chemistry Department (method of synthesis from patents WO 12073146 A1 and WO 10146488). All test compounds were $>95 \%$ pure, as determined by high-performance liquid chromatography (HPLC). Test compounds were prepared in sterile water to a dose volume of $2 \mathrm{ml} / \mathrm{kg}$ for subcutaneous injection. PF-04859989 is also commercially available via Sigma-Aldrich (St. Louis, MO).

\section{Bioanalytical}

KYNA from In Vivo Studies. Microdialysate samples from rat prefrontal cortex and CSF samples from NHPs were analyzed by online HPLC/fluorometric detection. KYNA was analyzed on a $150 \times$ 3-mm, 3- $\mu \mathrm{m}$ BDS Hypersil C18 column (Thermo Electron Corporation, Waltham, MA) set at $30^{\circ} \mathrm{C}$. The analytes were separated with a mobile phase containing $50 \mathrm{mM}$ sodium acetate ( $\mathrm{pH}$ to 6.3 with glacial acetic acid), $250 \mathrm{mM}$ zinc acetate, $2 \%-6 \%$ acetonitrile, delivered at $0.3-0.350 \mathrm{ml} / \mathrm{min}$ by an LC-10ADvp pump (Shimadzu, Columbia MD), and detected fluorometrically at Ex 344 /Em 398 nM (Shimadzu RFXL-10 detector). Extracellular levels of KYNA were measured and reported as nanomolar concentrations and percentage of baseline level, respectively. Data were collected by EZChrom Elite Client/ Server software version 3.2.1 (Agilent Technologies, Wilmington, DE).

KYNA from In Vitro Studies. KYNA levels of samples from in vitro KAT II turnover studies were quantified by liquid chromatography-tandem mass spectrometry (LC-MS/MS). The HPLC system consisted of a CTC PAL autosampler and Shimadzu LC20-AD pumps and degasser. Chromatography was achieved using a Phenomenex Synergi Polar-RP $(2.0 \times 50 \mathrm{~mm}, 4 \mu \mathrm{m}, 80 \AA)$ column. Mobile phase A consisted of $0.1 \%$ formic acid in HPLC water. Mobile phase B consisted of $0.1 \%$ formic acid in acetonitrile. The injection volume was $30 \mu \mathrm{l}$, and the flow rate was $0.350 \mathrm{ml} / \mathrm{min}$. The HPLC system was coupled to an Applied Biosystems (Mississauga, ONT) API 4000 mass spectrometer operated in positive multiple reaction monitoring mode. The transition monitored for kynurenic acid is $190.07>162.10$. The retention time for KYNA was 1.4 minutes; total run time was 3 minutes. The dynamic range of the assay was $0.010-5.0 \mathrm{ng} / \mathrm{ml}$. KYNA data acquisition, including peak integration and quantitation, were performed using Analyst 1.4.2. 


\section{Irreversible Inhibitors}

Concentrations of test compounds in rat brain and NHP CSF were measured using LC-MS/MS triple-quadrupole mass spectrometer. Assay details for PF-04859989 and PF-05182357 have been reported before (Tuttle et al., 2012). For PF-05579960 and PF-06253133, LC-MS/MS analysis was carried out using an HPLC system consisting of a Shimadzu LC-20 binary pump (Shimadzu Scientific Instruments) with a CTC PAL autosampler (Leap Technologies, Carrboro, NC) interfaced to an API 4000 LC-MS/MS quadrupole tandem mass spectrometer (Applied Biosystems/MDS Sciex Inc.). PF-06253133 and PF-05579960 were separated on an Analytical Sales and Service Advantage Echelon C18 column $(50 \times 2.1 \mathrm{~mm}, 4 \mu \mathrm{m})$ by gradient elution. Mobile phase A consisted of $5 \mathrm{mM}$ ammonium formate with $0.1 \%$ formic acid. Mobile phase B consisted of acetonitrile. The gradient began at a hold of $0 \% \mathrm{~B}$ for 0.3 minute and was then ramped up to $40 \%$ B over 2.2 minutes, immediately ramped to $90 \% \mathrm{~B}$, held there for 0.4 minutes, and then returned to $0 \% \mathrm{~B}$ for the remaining 0.6 minute of the method for re-equilibration. A flow rate of $0.45 \mathrm{ml} /$ min was used. The mass spectrometer was operated in positive-ion electrospray ionization mode for the detection of PF-06253133 and PF-05579960. Multiple reaction monitoring was performed with the transitions $m / z 204 \rightarrow 142.1$ for PF-06253133 and $m / z 253.1 \rightarrow 236.2$ for PF-05579960. All raw data were processed using Analyst Software version 1.4.2 (Applied Biosystems/MDS Sciex Inc.). The lower limit of quantification for PF-06253133 was $1.22 \mathrm{ng} / \mathrm{ml}$ and for PF-05579960, $0.61 \mathrm{ng} / \mathrm{ml}$. The upper limit of quantification was $2500 \mathrm{ng} / \mathrm{ml}$ for both compounds.

\section{In Vitro}

KAT II Turnover Assay. A whole-cell assay was developed to measure the recovery of enzyme activity after irreversible inhibition in relevant cells as a measure of turnover rate of KAT II enzyme. Test compounds were dissolved in 100\% dimethylsulfoxide (DMSO) and diluted to a working dilution in Hanks' balanced salt solution (HBSS).

Four types of cells from three species (detailed to follow) were cultured into six-well plates for each time point (three wells for maximal activity, three wells for activity with inhibitor) for eight time points between 0 and 72 hours.

1. Primary normal human astrocytes (provided frozen in a cryovial; Lonza Inc. Allendale, NJ) were thawed and seeded at $0.045 \times 10^{6}$ cells/well in a six-well plate on day 1 in astrocyte growth medium, which was replaced every other day. Cells were assay ready when close to $90 \%$ confluent, usually at day 8 to 9 .

2. Primary rat mixed glia cultures were obtained from rat cortices of P-2 Sprague-Dawley (Charles River, Raleigh, NC) rat pups per established protocols and under the Institutional Animal Care and Use Committee (IACUC) protocol 3389. Glia media consisted of DMEM high-glucose, $10 \%$ fetal bovine serum, $1 \%$ penicillin-streptomycin solution. The final cell suspension ( $\sim 1$ brain per $50 \mathrm{ml}$ in a T150 flask) was placed into culture on day 1 . On day 2 and approximately every 4 days after that, media were removed and replaced with fresh culture media. Flasks were confluent by days 11-14. At this point, typically, a confluent feeder layer of astrocytes with colonies of microglia was tethered to the astrocyte layer. Cells were harvested with trypsin and replated at $5 \times 10^{4}$ cells per well in six-well plates. Cells were fed with fresh media every other day and were assay-ready by day 8 , when they were confluent.

3. Primary rat astrocytes (provided frozen in a cryovial; Lonza Inc.) were thawed and seeded $0.045 \times 10^{6}$ cells per well in a six-well plate on day 1. AGM growth medium was replaced every other day. Cells were assay ready when close to $90 \%$ confluent, usually by week 3 .
4. Primary cynomolgus monkey hepatocytes (provided in a frozen cryovial; Bioreclamation IVT, Westbury, NJ) were plated into 96 -well plates at a concentration of $0.05 \times 10^{6}$ cells per well in a total volume of $200 \mu \mathrm{l}$ on day 1 (InvitroGro HI media; Bioreclamation IVT). On day 2, media were removed and replaced with HBSS; assay follows here.

Media were removed, and wells were washed once with HBSS (1 ml/ well of a six-well plate). The HBSS was removed and replaced with irreversible inhibitors diluted in HBSS or HBSS alone $(1 \mathrm{ml} / \mathrm{well}$ of a six-well plate). Plates were incubated for 1 hour at $37^{\circ} \mathrm{C}, 5 \% \mathrm{CO}_{2}$. The media, along with the inhibitors, were removed from all wells, and the wells were washed once with HBSS. For time points later than $t=0$, HBSS was replaced by complete media, and plates were returned to the incubator. For $t=0$, wells were washed once with ice-cold HBSS ( $1 \mathrm{ml} /$ well of a six-well plate). The substrate L-KYN was added to all wells ( $15 \mu \mathrm{M}$ for astrocytes or rat mixed glia, $1 \mathrm{ml} / \mathrm{well}$ of a six-well plate). The plates were incubated for 2 hours at $37^{\circ} \mathrm{C}, 5 \% \mathrm{CO}_{2}$. The supernatants $(850 \mu \mathrm{l} /$ well of a six-well plate) were removed and placed in a sample block. The samples were acidified with $100 \mu \mathrm{l}$ of $1 \mathrm{M} \mathrm{HCl}$. At the remaining time points, samples were washed once with HBSS, and the same procedure was followed as described for the $t=0$ time point. Samples were frozen at $-20^{\circ} \mathrm{C}$ until KYNA analysis by LC-MS/ MS.Percent inhibition was calculated based on maximal KYNA in wells not treated with irreversible inhibitors at each time point. The time courses of KAT II enzyme activity recovery from different cell types after irreversible inhibition were characterized by a turnover equation with zero-order KAT II synthesis rate $\left(K_{\text {syn }}^{K A T I I}\right)$ and first-order KAT II degradation rate $\left(k_{\text {deg }}^{K A T I I}\right)$ (Dayneka et al., 1993). KAT II turnover half-life was subsequently calculated for different cell types in eq. 1 and eq. 2 :

$$
\begin{aligned}
& \frac{d K A T I I}{d t}=K_{s y n}^{K A T I I}-k_{d e g}^{K A T I I} * K A T I I \\
& \text { KATII Half Life }=\operatorname{Ln}(2) / k_{d e g}^{K A T I I}
\end{aligned}
$$

Determination of Apparent $K_{m}$ for L-KYN for Recombinant KAT II. To characterize the kinetics of the recombinant KAT II enzymes in our laboratory and define assay conditions for subsequent $k_{\text {inact }} / K_{\mathrm{I}}$ assay, apparent $K_{\mathrm{m}}$ (the concentration of substrate that results in half-maximal reaction velocity under specified conditions) for L-KYN was determined. Initial conditions were based on those previously indicated (Schmidt et al., 1993). The assay was performed in 96-well or 384-well plates in a total volume of $150 \mu \mathrm{l}$ (96-well plate) or $30 \mu \mathrm{l}$ (384- well plate) per well. The assay mixture contained $70 \mu \mathrm{M}$ pyridoxal phosphate (PLP), $1 \mathrm{mM} \alpha$-ketoglutaric acid, and a fixed concentration (200 or $300 \mathrm{nM}$ ) of human, rat, or NHP KAT II in $150 \mathrm{mM}$ Tris acetate buffer, $\mathrm{pH} 7.0$, with $0.1 \%$ bovine $\gamma$-globulin. Substrate (L-KYN) was added to various final concentrations, from 0.05 to $3 \mathrm{mM}$. Enzyme concentration was chosen to allow the measurement of an initial linear rate across a large range of substrate values. Assay plates were sealed around the edges with tape and immediately read on a SpectraMax plate reader set at $37^{\circ} \mathrm{C}$ at an absorbance wavelength of $370 \mathrm{~nm}$. A $370 \mathrm{~nm}$ was recorded at 3-minute intervals for 4 hours.

Enzyme kinetic constants were determined by nonlinear regression analysis of a first-order Michaelis-Menten equation using GraphPad Prism 5.01 (GraphPad Software Inc., San Diego, CA). The apparent $K_{\mathrm{m}}$ values were expressed as millimolars. Arithmetic means and standard deviation of the $K_{\mathrm{m}}$ values were calculated from three experiments.

Estimation of $\boldsymbol{k}_{\text {inact }} / K_{\mathrm{I}}$ Using Recombinant KAT II. A stock solution of test compound was made in $100 \%$ DMSO and diluted to the required concentrations with the addition of $100 \%$ DMSO. An additional aqueous dilution was made so that the compound at $3 \times$ final concentration was $1.0 \%$ DMSO in the assay-specific buffer. Compounds were tested at 11 different concentrations. Final DMSO concentrations in the assay plate were equal to $0.33 \%$. 
KAT II enzyme activity after the addition of each test compound was followed by measuring the loss of absorbance of the L-KYN substrate at an absorbance wavelength of $370 \mathrm{~nm}$. The KAT II assays were run in a 384-well format at a final volume of $30 \mu \mathrm{l}$ using $150 \mathrm{mM}$ Tris Acetate buffer (pH 7.0), $1 \mathrm{mM}$ L-KYN, $1 \mathrm{mM} \alpha$-ketoglutaric acid, $70 \mu \mathrm{M}$ PLP, $0.1 \%$ bovine $\gamma$-globulin, and either $30 \mathrm{nM}$ human KAT II enzyme, $5 \mathrm{nM}$ rat KAT II enzyme, or $20 \mathrm{nM}$ NHP KAT II enzyme. Compound was diluted in 100\% DMSO and spotted before the addition of the other reagents. Enzyme was always added last. Assay plates were sealed around the edges with tape and immediately read on a SpectraMax plate reader at an absorbance wavelength of $370 \mathrm{~nm}$. The SpectraMax plate reader was set up to read every 5 minutes for 16 hours.

The following steps are taken to ensure generation of consistent kinetic data. 1) A 10- $\mu$ l aliquot of the compound dilutions (described here in compound preparation) was added to the assay plate by hand, followed by a quick spin to ensure the compound was collected at the bottom of the well; 2) a $10-\mu l$ aliquot of substrate mix containing the $L$ KYN ketoglutaric acid and PLP was then added to the assay plate via a multidrop instrument; 3) finally, a 10- $\mu$ l aliquot of a 3-fold concentration of enzyme stock solution was added last via a multidrop instrument to initiate the reaction; and 4) the microplate lid was placed onto the assay plate and taped to seal in humidity, and the assay plate was put into the SpectraMax reader. A quick vibration on the plate platform was done to ensure mixing, and the absorbance was read (wavelength of $370 \mathrm{~nm}$ ) every 5 minutes over 16 hours at room temperature.

DynaFit (BioKin Ltd., Geelong, Australia), a computational tool for mechanistic enzyme kinetics analysis (Kuzmic, 1996, 2009), was used to provide the global fit of the complete reaction progress curves. A previously established two-step model (Schwartz et al., 2014) assuming rapid equilibrium binding was implemented in estimating $k_{\text {inact }} / K_{\mathrm{I}}$ for each compound. KAT II $\mathrm{K}_{\mathrm{m}}$ for L-KYN was fixed to the appropriate value for rat, human, and NHP was determined from that step. The underlying system of first-order ordinary differential equations was integrated using the LSODE algorithm (Kuzmic, 1996, 2009).

Brain-Tissue Binding. Unbound fraction of the KAT II inhibitors was determined in rat brain tissue by standard equilibrium dialysis methods (Kalvass et al., 2007).

\section{In Vivo}

Rat. All procedures were carried out in compliance with the National Institutes of Health Guide for the Care and use of Laboratory Animals (1985), under approval of the IACUC.

In vivo microdialysis was performed in conscious, individually housed, freely moving male Sprague-Dawley rats (320-400 g; Charles River), which had free access to food and water and maintained on a 12-hour light/dark cycle. Microdialysis probe guide cannula (Bioanalytical Systems, Inc., West Lafayette, ID) were implanted in the prefrontal cortex (bregma: anteroposterior $+3.7 \mathrm{~mm}$, mediolateral $-0.7 \mathrm{~mm}$, dorsoventral $-2.0 \mathrm{~mm}$ ) (Paxinos and Watson, 1982) under isoflurane anesthesia and fixed to the skull using bone screws and dental acrylic. Animals recovered for a minimum of 24 hours. Approximately 18 hours before testing, guide stylets were removed, and probes (BR-4; Bioanalytical Systems, Inc.) with a polyacrylonitrile membrane (outside diameter, $320 \mu \mathrm{m}$; MW cutoff, 30,000 Da) and a 4-mm dialysis tip were inserted. The inlet and outlet of the probe were connected with flexible polyetheretherketone tubing (inside diameter, 0.005 inch; Upchurch Scientific, Vernon Hills, IL), and the probes were perfused at $(1.5 \mu \mathrm{l} / \mathrm{min})$ with artificial CSF (147 mM NaCl, $2.7 \mathrm{mM}$ $\mathrm{KCl}, 1.0 \mathrm{mM} \mathrm{MgCl}$ hexahydrate, $1.3 \mathrm{mM} \mathrm{CaCl}{ }_{2}$ dihydrate) with a CMA/100 (CMA/Microdialysis, North Chelmsford, MA) microperfusion pump. On the day of testing, microdialysate samples (30 $\mu \mathrm{l})$ were continuously collected and automatically injected every 20 minutes for KYNA analysis by online HPLC/fluorometric detection.

For systemic administration, rats were dosed subcutaneously with vehicle $(2 \mathrm{ml} / \mathrm{kg}$ ) or compound dissolved in vehicle. Vehicle consisted of
$1 \%$ acetic acid in distilled water volume to volume. Compound or vehicle was administered subcutaneously approximately 2 hours after basal KYNA levels stabilized, and the effects on KYNA levels were monitored for at least 18 hours. PF-04859989 was dosed s.c. at 3.2, 5.6, 10,32 , and $100 \mathrm{mg} / \mathrm{kg}(n=3-14)$. Both PF-05579960 and PF-06253133 were dosed s.c. at $1,3.2,10$, and $32 \mathrm{mg} / \mathrm{kg}(n=3)$. Compound exposures in the rat brain were determined from satellite animals $(n=$ 2 to 3). PF-04859989 brain samples were collected at $0.25,0.5$, and 1 hour after $3.2 \mathrm{mg} / \mathrm{kg}$ s.c.; $0.25,0.5,1$, and 3 hours after administration of $10 \mathrm{mg} / \mathrm{kg}$ s.c.; $0.25,0.5,1,2$, and 4 hours after $32 \mathrm{mg} / \mathrm{kg}$ s.c.. PF-05579960 and PF-06253133 brain samples were collected at $0.5,1,2,4$, and 7 hours after s.c. $5 \mathrm{mg} / \mathrm{kg}$. All brain samples were homogenized in 60/40 isopropanol/water with a magnetic bead beater, centrifuged. Fifty milliliters of supernatant was precipitated with $300 \mu \mathrm{l}$ of acetonitrile containing internal standard, vortexed, and centrifuged. The supernatant was subsequently dried under nitrogen and reconstituted in $80 \mu \mathrm{l}$ of $95 / 5$ water/acetonitrile. Five microliters was then injected on the LC-MS/MS for analysis.

NHP [Cynomolgus macaques (Macaca fascicularis)]. This study was approved by the IACUC of Maccine under protocol number 46-2007 before the start of the experimental phase. Ten NHP [Pusat Studi Satwa Primate (Primate Research Center), Institut Pertanian Bogor, Indonesia] aged 3-5 years old were used in this study (two males and eight females). Animals were housed singly under 12-hour light/dark cycle. During the study, the cages were cleaned at regular intervals to maintain hygiene. NHPs were surgically prepared with indwelling cannulas inserted into the cisterna magna and connected to a subcutaneous access port to permit CSF sampling. Animals were allowed a minimum 7-day recovery period from surgery before the commencement of dosing and sampling. The animals were restrained in primate chairs on each day of dosing. On the day of dosing, PF-05182357 was administered subcutaneously with a dose volume of $1.0 \mathrm{ml} / \mathrm{kg}$ at 1 and $3 \mathrm{mg} / \mathrm{kg}(n=5)$. Both blood and CSF samples were collected at the following time points in relation to dosing: day -2 (-48), day -1 (-24), 0, 0.25, 0.5, 1, 2, 4, 7, 12, 24, 48, 72, and 120 hours. Whole-blood samples of approximately $1.0 \mathrm{ml}$ were collected from the femoral vein and into EDTA-containing tubes. Plasma was separated by spinning the samples at $4000 \mathrm{rpm}$ for 10 minutes at $4^{\circ} \mathrm{C}$. For collection of the CSF samples via the cisterna magna catheter, the area around the port was swabbed with a suitable disinfectant before insertion of the Huber needle. After needle insertion, a 150- $\mu$ l sample was taken and discarded, and then a $300-\mu l$ sample was obtained. Each $300-\mu \mathrm{l}$ sample was in $3 \times 100-\mu \mathrm{l}$ aliquots in cryovials and snapfrozen on liquid nitrogen. Both plasma and CSF samples were stored at $-80^{\circ} \mathrm{C}$ before analysis. Both PF-05182357 and KYNA concentrations were subsequently measured from CSF samples; only PF-05182357 concentration was measured in plasma samples.

\section{Data Analysis}

Rat PKPD Modeling. A sequential PKPD analysis (Zhang et al., 2003) was performed: 1) the PK profiles of PF-04859989, PF-05579960, and PF-06253133 from satellite groups were characterized independently; and 2) the PD responses of PF-04859989, PF-05579960, and PF-06253133 were simultaneously modeled while fixing PK parameters estimated from step 1. The PK profiles of PF-04859989 (step 1) were characterized using a standard two-compartment model with zeroorder absorption and first-order elimination. For PF-05579960 and PF-06253133, a time-lag two-compartment model (Singh et al., 1988) was applied to capture the second absorption peak.

The PD response was represented as the percentage of baseline KYNA levels, which was defined as the average KYNA concentrations over 80 minutes before dosing. The PKPD model (Fig. 2) that mechanistically characterizes the irreversible inhibition of KAT II enzyme was adapted from a previously published model (Hutmacher et al., 2005). Briefly, zero-order synthesis rates and first-order degradation rates were assumed for both KAT II and KYNA to maintain their steady-state levels under normal physiologic conditions 


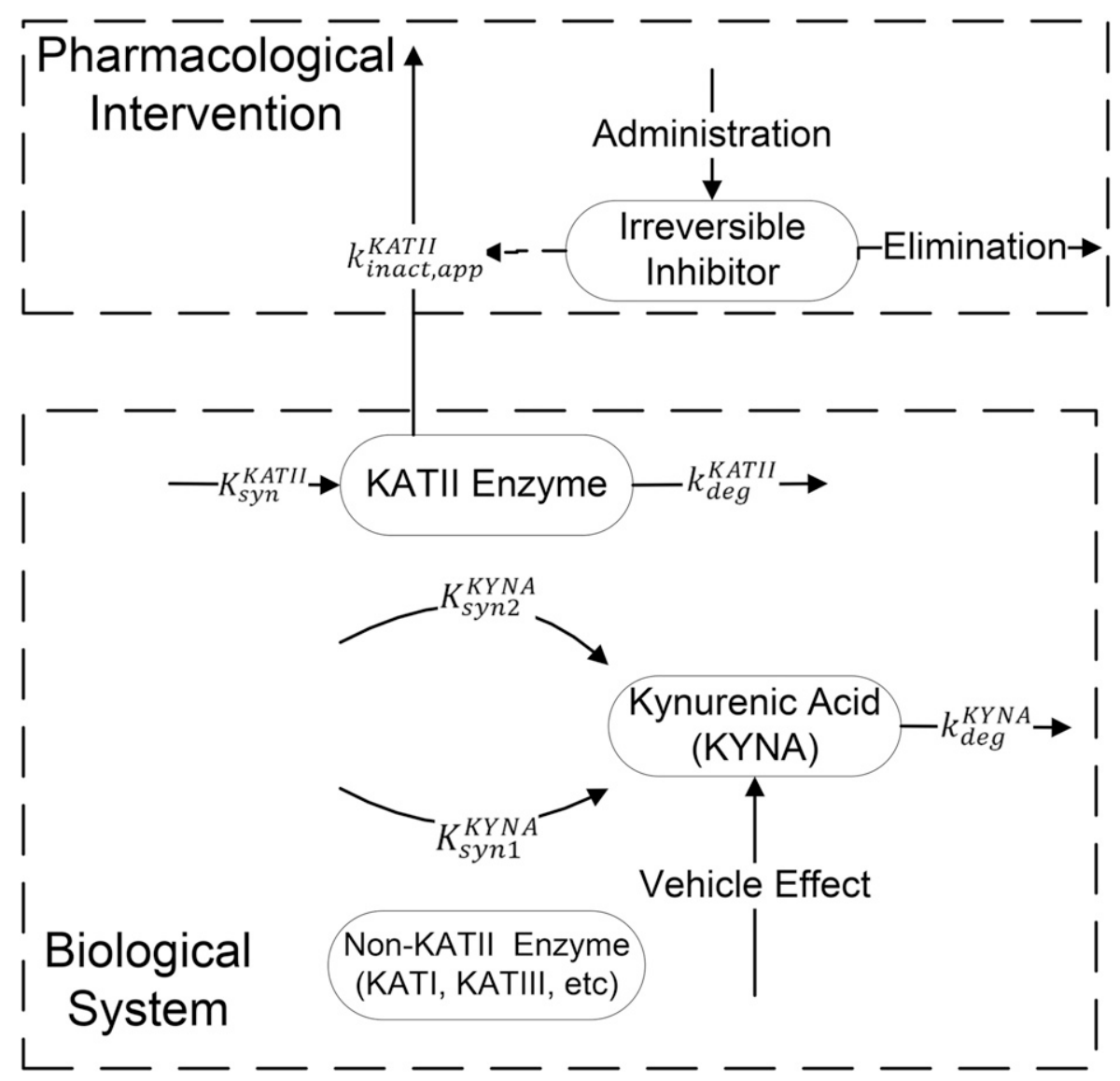

Fig. 2. Mechanism-based PK-PD model structure. $K_{s y n}^{K A T I I}, K_{s y n 1}^{K Y N A}$ and $K_{\text {syn }}^{K Y N}$ represent the synthesis rates for KAT II, KYNA (via nonKAT II), and KYNA (via KAT II), respectively. $k_{\text {deg }}^{K A T I I}$ and $k_{\text {des }}^{K Y N A}$ represent the degradation rates for KAT II and KYNA, respectively. $k_{\text {inoct }}^{K Y p p}$ represents the inactivation rate of KAT II by irreversible inhibitors.

(Dayneka et al., 1993). Administration of an irreversible KAT II inhibitor effectively creates a secondary elimination pathway for the enzyme, which is directly linked to the inhibitor concentration. Clearance of the inhibitor from the circulation relieves the drain on KAT II, thus allowing it to recover to previous physiologic level. It is challenging to monitor active enzyme levels over the course of pharmacologic intervention; instead, the product KYNA is monitored as a surrogate of KAT II enzyme activity. More specifically, the model is described as in eq. $3-6$ :

$$
\begin{aligned}
& \frac{d K Y N A}{d t}=K_{\text {syn1 }}^{K Y N A}+K_{\text {syn2 }}^{K Y N A} \times K A T I I-k_{\text {deg }}^{K Y N A} \times K Y N A \\
& \frac{d K A T I I}{d t}=K_{\text {syn }}^{K A T I I}-\left[k_{\text {deg }}^{K A T I I}+k_{\text {inact }, \text { app }}^{K A T I I}\right] \times K A T I I
\end{aligned}
$$

where

$$
k_{\text {inact }, a p p}^{\text {KATII }}=\left(\frac{k_{\text {inact }}^{\text {KATII }}}{K_{I}+[I]}\right) \times[I]
$$

Or when $[I]$ is much lower than $K_{I}$,

$$
k_{\text {inact }, a p p}^{\text {KATII }}=\left(\frac{k_{\text {inact }}^{\text {KATII }}}{K_{I}}\right) \times[I],
$$

where $K_{\text {syn }}^{K A T I I}$ is the zero-order synthesis rate of the KAT II enzyme, $k_{\text {deg }}^{K A T I I}$ is the first-order degradation rate of the KAT II enzyme, $k_{\text {inact,app }}^{K Y N A}$ represents the inactivation of KAT II by irreversible inhibitors $[I], K_{s y n 1}^{K Y N A}$ is the zero-order synthesis rate of KYNA by non-KAT II enzymes, and $K_{\text {syn } 2}^{K Y N A}$ is the first-order synthesis rate of KYNA by KAT II, $k_{\text {deg }}^{K Y N A}$ is the first-order degradation rate of KYNA. The substrate $\mathrm{L}-\mathrm{KYN}$ is assumed to be in excess and thus constant. Both the complete inactivation function (eq. 5) and the simplified linear function (eq. 6) were used to fit experimental data. Steady-state KAT II enzyme level was not measured and hence set at the nominal value of 1 . KYNA steady-state level was fixed at $100 \%$ since all observations are normalized by predose baseline. Vehicle effect on KYNA was observed from vehicle-treated rats, possibly owing to stress, and was modeled as a stimulatory effect on KYNA with bolus input function, followed by first-order elimination. Time courses of KYNA levels after administering three different irreversible inhibitors at different doses were simultaneously fitted according to the PKPD model in estimating one set of system parameters (i.e., $K_{s y n 1}^{K Y N A}, K_{s y n 2}^{K Y N A}, k_{d e g}^{K Y N A}, K_{s y n}^{K A T I}, k_{d e g}^{K A T I I}$ ) and compound-dependent in vivo potency parameters (i.e., $k_{\text {inact }}^{\text {KATII }}, K_{I}$ ).

Nonhuman Primate PKPD Modeling. Since both drug concentrations and KYNA responses were collected from the same animals, a population PKPD model was developed to characterize the relationship between PF-05182357 concentration and KYNA response within individuals. A sequential PKPD modeling approach similar to the rat model was adopted. PF-05182357 plasma concentrations were first characterized using a standard three-compartment model with zeroorder absorption and first-order elimination. Interindividual variability on different PK parameters was evaluated for best individual fit. Subsequently, individual PK parameters were fixed in estimating population $\mathrm{PD}$ parameters based on the same model structure as used in the rat analysis. PD response was represented as the percentage of baseline KYNA levels, defined as the average KYNA concentrations between -24 and 0 hour.

Model Fitting and Evaluation. The PKPD data were modeled using nonlinear mixed-effects modeling as implemented in the NONMEM (NONMEM software system, version 7.2; GloboMax LLC, Hanover, MD). Log transformation was applied to exposure measurements before analysis. Proportional residual error model was used for all models. The analyses were conducted using the first-order conditional estimation with interaction method. Ninety percent 

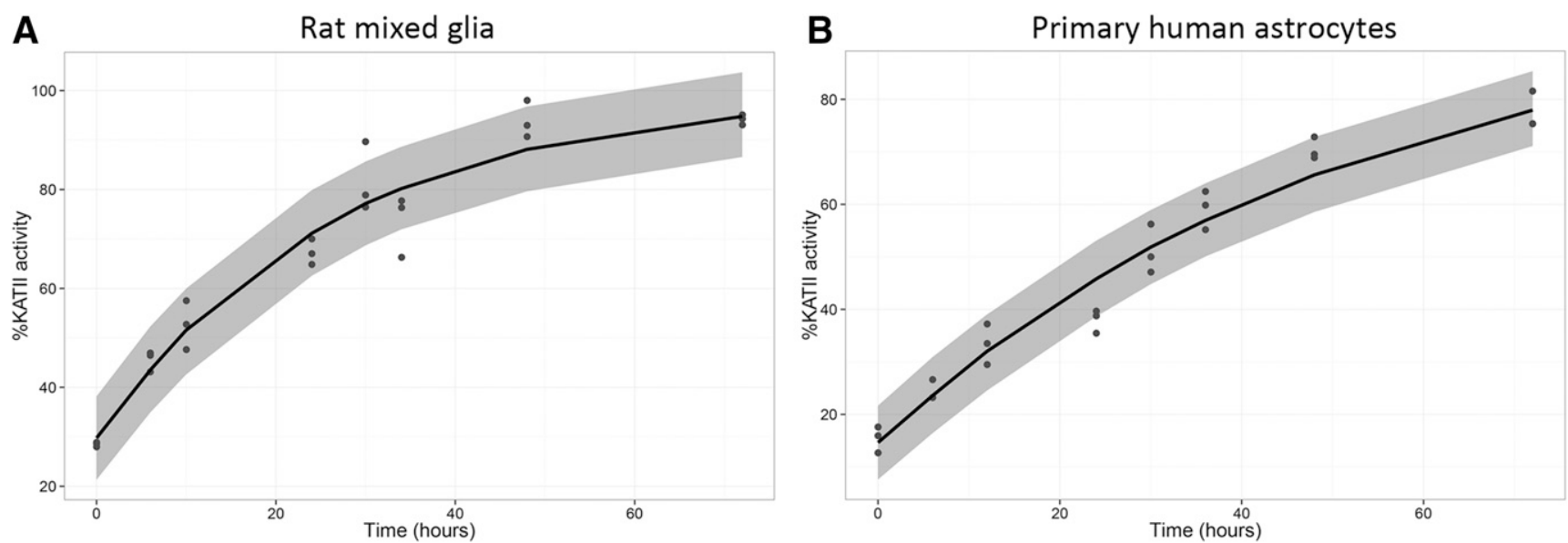

Fig. 3. Comparison of model-simulated time courses of KAT II activity recovery after irreversible inhibition in rat mixed glia (A) and primary human astrocytes (B) to corresponding observations. Solid circles represent the observed data, solid lines represent the simulated median, and shaded area represents the 5th and 95th percentiles from model prediction.

confidence intervals (CI) for the parameter estimates in the final model were estimated using the parametric bootstrap approach. Graphical data display was done using R.2.15.3.

Assessment of the model goodness-of-fit was conducted based on the following standard methods. Models were evaluated using the following goodness-of-fit criteria: successful minimization of the objective function, visual inspection of several diagnostic scatter plots (population and individual observed vs. predicted concentrations, residual plots, plots of random effects, and weighted residuals vs. time, histograms of individual random effects), change in the objective function value relative to the change in number of parameters, as well as the magnitude and precision of the parameter estimates.

\section{Results}

KAT II Turnover Assay. The addition of various KAT II inhibitors (i.e., PF-04859989 or PF-05182357) in the four different whole-cell assays resulted in an initial inhibition of approximately $50 \%-80 \%$ of KAT II activity. Over time, enzyme activity gradually returned as the processes of KAT II enzyme synthesize and its subsequent degradation reached steady state under noninhibited conditions. The time courses of enzyme activity recovery in cells from different tissues were adequately described by the zero-order production first-order degradation model (Fig. 3). The estimated KAT II turnover half-life is similar in human astrocytes and NHP hepatocytes (43.4 and 34.7 hour, respectively). In primary rat mixed glia and primary astrocytes, the average KAT II half-life is much shorter at 14.9 hours. Model-fitted parameters are listed in Table 1.

Brain Tissue Binding. Similar rat brain tissue unbound fractions of PF-04859989, PF-05579960, and PF-06253133 were estimated $(0.39,0.29$, and 0.47 , respectively) from the equilibrium dialysis method.

Determination of Apparent $K_{\mathrm{m}}$ for L-KYN for Recombinant KAT II. A simple absorbance method to measure KAT II activity was developed and used to determine the apparent $K_{\mathrm{m}}$ for L-KYN for human, rat, and rhesus monkey KAT II enzymes. $K_{\mathrm{m}}$ values derived for all three species are comparable (Table 2 ) and consistent with the $K_{\mathrm{m}}$ values reported in the literature for rat KAT II $(0.88 \mathrm{mM}$; Guidetti et al., 1997) and human KAT II (2.5 mM; Schmidt et al., 1993). Similar $K_{\mathrm{m}}$ values were also reported from a more extensive cross-species investigation of KAT II (Lu et al., 2016).

Estimation of $\boldsymbol{k}_{\text {inact }} / \boldsymbol{K}_{\mathrm{I}}$ Using Recombinant KAT II. The kinetic rate data were obtained by measuring loss of absorbance for the substrate L-KYN. A representative set of A $370 \mathrm{~nm}$ versus time curves at different dilutions of PF-06253133 in the human KAT II assay is shown in Fig. 4. A reasonably linear loss of absorbance was observed over 16 hours in the absence of PF-06253133. In the presence of PF-06253133, the progress curves for the KAT II reaction exhibited a curvature (Fig. 4), which is consistent with the mechanism of irreversible inhibition. Both $k_{\text {inact }}$ and $K_{\mathrm{I}}$ were successfully estimated for all four irreversible inhibitors in recombinant rat/NHP and human KAT II assays. To compare with the corresponding in vivo potency estimation, the apparent second-order rate constant potency estimates $\left(k_{\text {inact }} / K_{\mathrm{I}}\right)$ were subsequently calculated and are listed in Table 1.

Rat PKPD Modeling. After subcutaneous administration in rats, all three tested compounds demonstrated rapid absorption with brain exposure peaks at the first time point (0.25-0.5 hour) and were quickly eliminated with half-lives of less than 1 hour. Their PK profiles were sufficiently characterized using the proposed PK models, which were subsequently used to drive PD interactions with KAT II enzymes (Fig. 5). Significant vehicle effect was observed in KYNA responses, potentially owing to the stress effect of subcutaneous dosing, in which a transient increase in the KYNA level was followed by slow dissipation over the course of observation. Brain KYNA levels (PD response) decreased dose responsively after the administration of irreversible inhibitors. A maximum of $80 \%$ KYNA decrease was observed at approximately 2-4 hours postdosing for all three inhibitors. KYNA levels slowly recovered over the course of 24 hours. Apparent temporal disconnect between PK and PD profiles was observed where the onset of PD response is delayed, and the duration of PD response is prolonged compared with those of the PK profile. This observation is consistent with irreversible inhibition of a target that has slow turnover kinetics. The initial complete model (eq. 5) failed to identify independently $k_{\text {inact }}$ and $K_{I}$ because of the high correlation between the two parameters, suggesting that the current exposure response relationship could be at a linear range. The simplified linear 
TABLE 1

Compound and biologic system parameter estimates from in vitro experiments

Mean values are expressed as arithmetic means.

\begin{tabular}{|c|c|c|c|c|c|c|}
\hline Parameter Type & Compound/System & Parameter & Species & Estimate & S.D. & $\mathrm{n}$ \\
\hline \multirow[t]{11}{*}{ Compound-specific } & PF-04859989 & $k_{\text {inact }} / K_{I}\left(\mathrm{M}^{-1} \mathrm{~s}^{-1}\right)$ & Rat & 134 & 9 & 10 \\
\hline & & & Human & 6239 & 2279 & 13 \\
\hline & & $\mathrm{Fu}, \mathrm{b}$ & Rat & 0.39 & $\mathrm{n} / \mathrm{a}$ & 1 \\
\hline & PF-05579960 & $k_{\text {inact }} / K_{I}\left(\mathrm{M}^{-1} \mathrm{~s}^{-1}\right)$ & Rat & 564 & $536,591^{a}$ & 2 \\
\hline & & & Human & 5985 & 2091 & 4 \\
\hline & & $\mathrm{Fu}, \mathrm{b}$ & Rat & 0.29 & $0.28,0.30^{a}$ & 2 \\
\hline & PF-06253133 & $k_{\text {inact }} / K_{I}\left(\mathrm{M}^{-1} \mathrm{~s}^{-1}\right)$ & Rat & 477 & 28 & 6 \\
\hline & & & Human & 20,032 & 5997 & 8 \\
\hline & & $\mathrm{Fu}, \mathrm{b}$ & Rat & 0.47 & $0.23,0.71^{a}$ & 2 \\
\hline & PF-05182357 & $k_{\text {inact }} / K_{I}\left(\mathrm{M}^{-1} \mathrm{~s}^{-1}\right)$ & Monkey & 16,668 & $18,689,14,646^{a}$ & 2 \\
\hline & & & Human & 13,895 & 768 & 4 \\
\hline \multirow[t]{3}{*}{ System-specific } & KAT II & Half-life (h) & $\mathrm{Rat}^{b}$ & 15 & 4.3 & 4 \\
\hline & & Half-life (h) & Human $^{c}$ & 44 & $36,52^{a}$ & 2 \\
\hline & & Half-life (h) & Monkey $^{d}$ & 35 & $\mathrm{n} / \mathrm{a}$ & 1 \\
\hline
\end{tabular}

Fu,b, Fraction unbound in brain tissue; KAT II, kynurenine aminotransferase II; $K_{\mathrm{I}}$, concentration of inactivator that yields $1 / 2 k_{\text {inact }} ; k_{\text {inact }}$, inhibition constant defined as the maximum rate of inactivation at infinite inhibitor concentration; $\mathrm{n} / \mathrm{a}$, not applicable.

${ }^{a}$ Individual parameter estimates were listed when $n$ is less than three.

${ }^{b}$ From primary rat mixed glia and astrocytes.

${ }^{c}$ From primary human astrocytes.

${ }^{d}$ From primary monkey hepatocytes.

model (eq. 6) successfully converged and sufficiently characterized the pharmacodynamic profiles of the three inhibitors at all dose levels (Fig. 5). All model parameters were estimated with sufficient certainty except the KYNA turnover rate $\left(k_{d e g}^{K Y N A}\right)$, which was estimated to be a large number with high uncertainty on the initial run. This finding suggests that KYNA is rapidly turned over, and the current data set is not sufficient to pinpoint the exact rate. Consequently, $k_{d e g}^{K Y N A}$ was fixed to 1000 hour $^{-1}$ (corresponds to a half-life of 2.5 seconds). The model estimated that KAT II enzyme contributes to $75 \%$ of KYNA formation, whereas non-KAT II enzymes are responsible for the remaining production. Rat brain KAT II turnover half-life was estimated to be 15.5 hours (Table 3 ).

After correcting for brain tissue binding, PF-06253133 and PF-05579960 were estimated to be more potent than PF-04859989 with free concentration-based $k_{\text {inact }} / K_{\mathrm{I}}$ of 848.6, 535.4, and 80.6 $\mathrm{M}^{-1} \mathrm{~s}^{-1}$, respectively (Table 3).

Nonhuman Primate PKPD Modeling. Although PF$05182357 \mathrm{CSF}$ concentrations were undetectable for all time points except the first two $(0.25$ and 0.5 hour $)$ owing to its impaired brain penetration and rapid clearance, significant and dose-responsive CSF KYNA reduction was observed, suggesting its potent inhibitory effect at KAT II. This is consistent with its potent in vitro activity $\left(k_{\text {inact }} / K_{\mathrm{I}}=\right.$ $\left.16,668 \mathrm{M}^{-1} \mathrm{~s}^{-1}\right)$. KYNA levels slowly recovered over the entire observation period (5 days). To characterize fully the PK profile and understand better KATII enzyme turnover, plasma concentrations were used to support PKPD analysis. Individual PF-05182357 plasma concentration-time profiles were sufficiently captured by a three-compartment model with interindividual variability on relative availability and intercompartmental clearance (Fig. 6). The proposed PKPD model adequately characterized the time course of PF-05182357-induced KYNA reduction response (Fig. 6). PF-05182357 $k_{\text {inact }} / K_{\mathrm{I}}$ was estimated to be $548.3 \mathrm{M}^{-1} \mathrm{~s}^{-1}$ based on total plasma concentration. The difference between in vivo $k_{\text {inact }} / K_{\mathrm{I}}$ and in vitro $k_{\text {inact }} / K_{\mathrm{I}}$ for PF-05182357 is most likely due to impaired brain penetration where total plasma concentration is much higher than unbound brain concentration. KAT II turnover half-life was estimated to be 76 hours.
Because of limited observations from the NHP study and high variability, the CIs for both parameters were large. Also, in contrast to the rat model, where KYNA half-life was too short to be determined, KYNA turnover half-life was successfully estimated to be 1 hour for NHP, suggesting that the KYNA turnover rate is slower in this species. In NHP, KAT II was estimated to contribute to $86 \%$ of KYNA formation (Table 3 ).

\section{Discussion}

KYNA has important physiologic roles in human brain function, and its elevation has been hypothesized to be causal to cognitive impairment in multiple central disorders, including schizophrenia. Development of a safe and efficacious pharmaceutical agent to test this hypothesis in humans will provide the ultimate answer. KAT II is considered the most plausible target for pharmacologic modulation of brain KYNA levels and has been extensively characterized (Rossi et al., 2008; Passera et al., 2011). Our modeling results quantitatively support this finding. As all irreversible inhibitors tested in this report are at least 50-fold selective against KAT I or KAT III, and no KAT IV inhibition was observed at up to $50 \mu \mathrm{M}$ PF-04859989 (internal data), achievement of $\sim 80 \%$ maximal KYNA inhibition in both rats and NHPs with these inhibitors suggests a dominant role for KAT II in brain KYNA formation. More quantitatively, the model estimated KAT II contribution to brain KYNA formation in rats and NHPs to be $75 \%$ and $86 \%$, respectively. This confirms previous findings that KAT II catalyzes approximately $75 \%$ of KYNA synthesis

\section{TABLE 2}

$\mathrm{K}_{\mathrm{M}}$ values for L-KYN of different KAT II enzymes Values are expressed as arithmetic means.

\begin{tabular}{lcc}
\hline \multicolumn{1}{c}{ Assay } & $\mathrm{K}_{\mathrm{M}}, \mathrm{mM} \pm$ S.D. & $n$ \\
\hline Human KAT II & $1.11 \pm 0.07$ & 3 \\
Rat KAT II & $1.50 \pm 0.28$ & 3 \\
Rhesus monkey KAT II & $1.44 \pm 0.09$ & 3
\end{tabular}

KAT II, kynurenine aminotransferase II; $K \mathrm{~m}$, the concentration of substrate that results in half-maximal reaction velocity. 


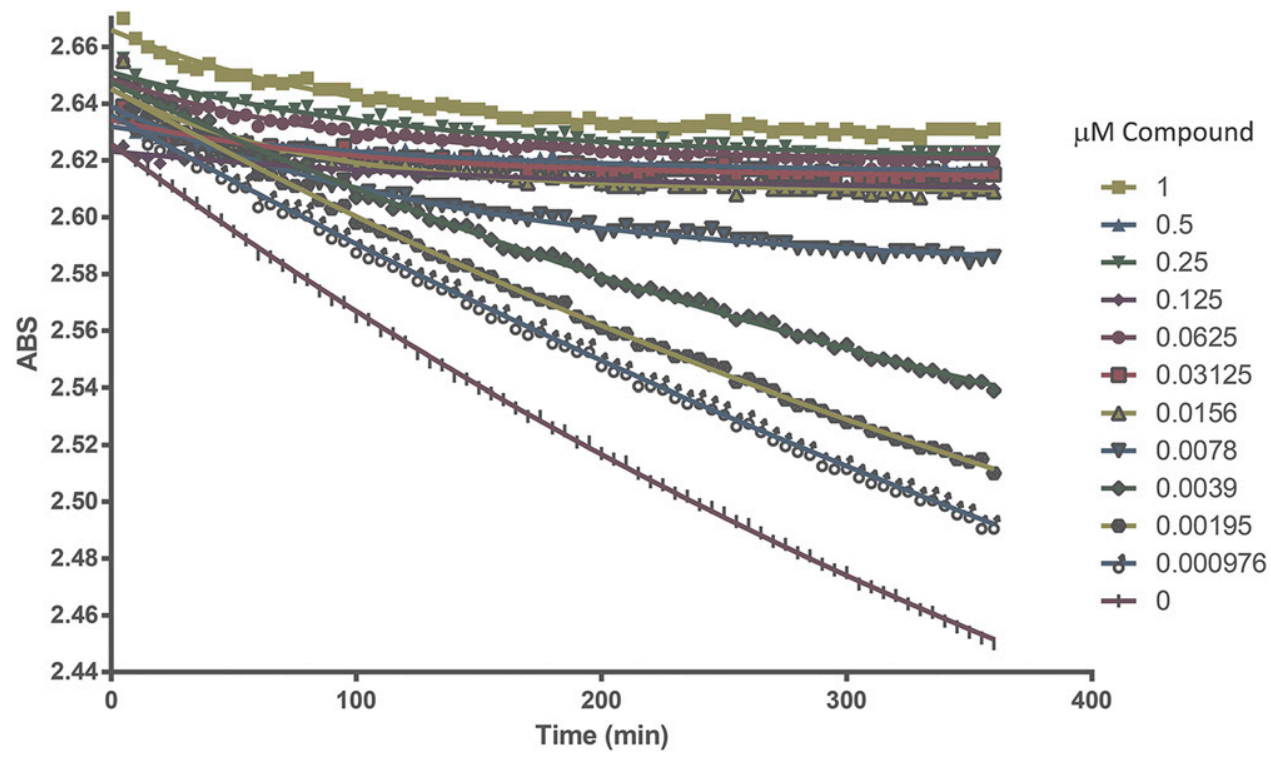

Fig. 4. Representative reaction progress curves for human KAT II to estimate the in vitro $k_{\text {inact }} / K_{\mathrm{I}}$. The depletion of $\mathrm{L}-\mathrm{KYNA}$ (represented by the loss of absorbance at $370 \mathrm{~nm}, \mathrm{ABS}$ ) in the presence of different levels of PF-06253133 is plotted.

in most brain areas (Guidetti et al., 1997). More recently, the specific contribution of KAT IV to KYNA synthesis was studied and reported to be very limited (Guidetti et al., 2007). These findings further validate KAT II as a viable target for effective lowering of brain KYNA.

Despite the significance of KAT II as an attractive target, no estimation of KAT II turnover properties has been reported to date. This is an important target property that could affect the duration of therapeutic effect elicited by pharmacologic agents, especially irreversible inhibitors. In the current study, this property was thoroughly investigated in rats and NHPs in vivo as well as in rat, NHP, and human in vitro systems. There is general agreement between estimations from in vitro and in vivo systems. Both systems suggest that KAT II turnover half-life is longer in NHPs or humans than in rodents. Specifically, KAT II half-lives in rats and NHPS in vivo system were estimated to be 15.5 and 76.3 hours, respectively. Similarly, KAT II half-lives in rat, NHP, and human primary cells were estimated to be $14.9,34.7$, and 43.4 hours, respectively. In vitro estimated KAT II half-life is

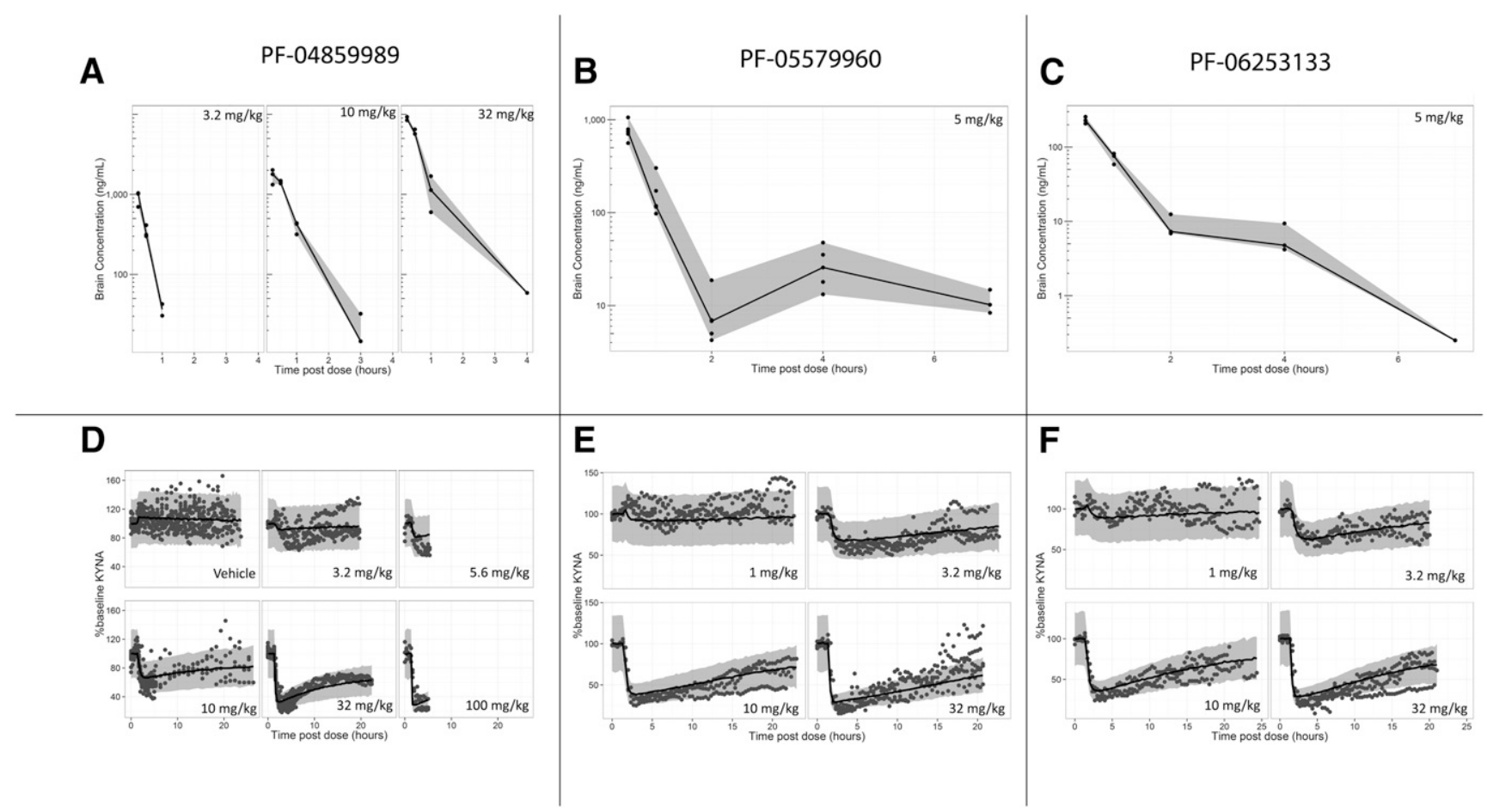

Fig. 5. Comparison of model-simulated time courses of irreversible inhibitor brain concentrations: (A) PF-04859989, (B) PF-05579960, (C) PF-06253133), and brain KYNA-lowering effects (D) PF-04859989, (E) PF-05579960, (F) PF-06253133) to corresponding observations in rats. Solid circles represent observed data, solid lines represent the simulated median, and shaded area represents the 5th and 95th percentiles from model prediction. 
TABLE 3

Compound and biologic system parameter estimates from in vivo experiments

\begin{tabular}{cccccc}
\hline Parameter Type & Compound/System & Parameter & Species & Estimate & $90 \%$ CI \\
\hline Compound-specific & PF-04859989 & $k_{\text {inact }} / K_{\mathrm{I}}\left(\mathrm{M}^{-1} \mathrm{~s}^{-1}\right)$ & Rat & 80.6 & $(17.1,89.8)$ \\
& PF-05579960 & $k_{\text {inact }} / K_{I}\left(\mathrm{M}^{-1} \mathrm{~s}^{-1}\right)$ & Rat & 535.4 & $(123.6,603.4)$ \\
& PF-06253133 & $k_{\text {inact }} / K_{I}\left(\mathrm{M}^{-1} \mathrm{~s}^{-1}\right)$ & Rat & 848.6 & $(170.4,991)$ \\
System-specific & PF-05182357 & $k_{\text {inact }} / K_{I}\left(\mathrm{M}^{-1} \mathrm{~s}^{-1}\right)$ & Monkey & 548.3 & $(421.5,14,850)$ \\
& KAT II & Half-life $(\mathrm{h})$ & Rat & 15.5 & $(14.8,16.2)$ \\
& KYNA & Half-life (h) & Rat & $0.001^{a}$ & $\mathrm{n} / \mathrm{a}$ \\
& KAT II & KAT II contribution $(\%)$ & Rat & 74.9 & $(73.4,100)$ \\
& KYNA & Half-life (h) & Monkey & 76.3 & $(6.36,118.9)$ \\
& & Half-life (h) & Monkey & 0.97 & $(0.70,1.5)$
\end{tabular}

CI, Confidence interval; KAT II, kynurenine aminotransferase II; $K_{I}$, Concentration of inactivator that yields $1 / 2 k_{\text {inact }}$ $k_{\text {inact }}$, Inhibition constant is defined as the maximum rate of inactivation at infinite inhibitor concentration; KYNA kynurenic acid; n/a, not applicable.

${ }^{a}$ Parameter is fixed.

highly predictive of in vivo estimation for rat (14.9 hours vs. 15.5 hours) and to a lesser extent for NHPs (34.7 hours vs. 76.3 hours). This discrepancy could be the result of NHP in vivo KAT II half-life being poorly estimated (90\% confidence interval ranges from 6.4 to 118.9 ) from sparse and variable NHP observations, which contrasts to high confidence estimation in rats (90\% CI ranges from 14.8 to 16.2), where extensive observations from multiple compounds were modeled simultaneously. Based on overall consistent in vitro-in vivo correlation (IVIVC), human in vivo KAT II half-life is projected to be approximately 43 hours. Slow KAT II turnover rate, especially in NHPs and humans, greatly enhanced the duration of the KYNA-lowering effect from irreversible inhibition (i.e., the KYNA level remains low even after circulating irreversible inhibitor has been cleared from the system). To illustrate this effect, the human KYNA-lowering response after administering PF-06253133, was simulated (Fig. 7A). For this simulation, in addition to human KAT II half-life, PF-06253133 in vivo potency was also scaled to human based on established potency IVIVC (vide infra). Additionally, projected PF-06253133 human PK parameters (not reported) were also used to best represent clinical response. As illustrated in Fig. 7A, although PF-06253133 was projected to have a PK half-life that is too short to sustain once-a-day dosing, where most drug is cleared from the system within 10 hours, the PD effect is much prolonged. It takes $\sim 5$ days for the PD effect to fully develop. This finding significantly accelerated the drugdiscovery process by opening the chemical space to compounds with a short half-life, which is typically excluded from clinical candidate selection. A short half-life clinical candidate also has an added safety advantage because of lower overall body burden, and hence less undesirable side effects. Another hypothetical simulation assuming a much shorter KAT II half-life of 1 hour is performed (Fig. 7B). Under this condition, the steady-state KYNA-lowering effect is significantly reduced from the same dose of PF-06253133. More importantly, PD response now tracks the $\mathrm{PK}$ profile, resulting in increased KYNA variation (i.e., peak to trough ratio) within each dosing period, especially when KAT II inhibitor is given at higher doses. This type of KYNA-lowering profile could be detrimental to mental health because of the significant role of KYNA in brain function. Target turnover kinetics has been routinely evaluated for biotherapeutic drugs (Luu et al., 2012; Zhao et al., 2012); however, such investigation for small-molecule drug
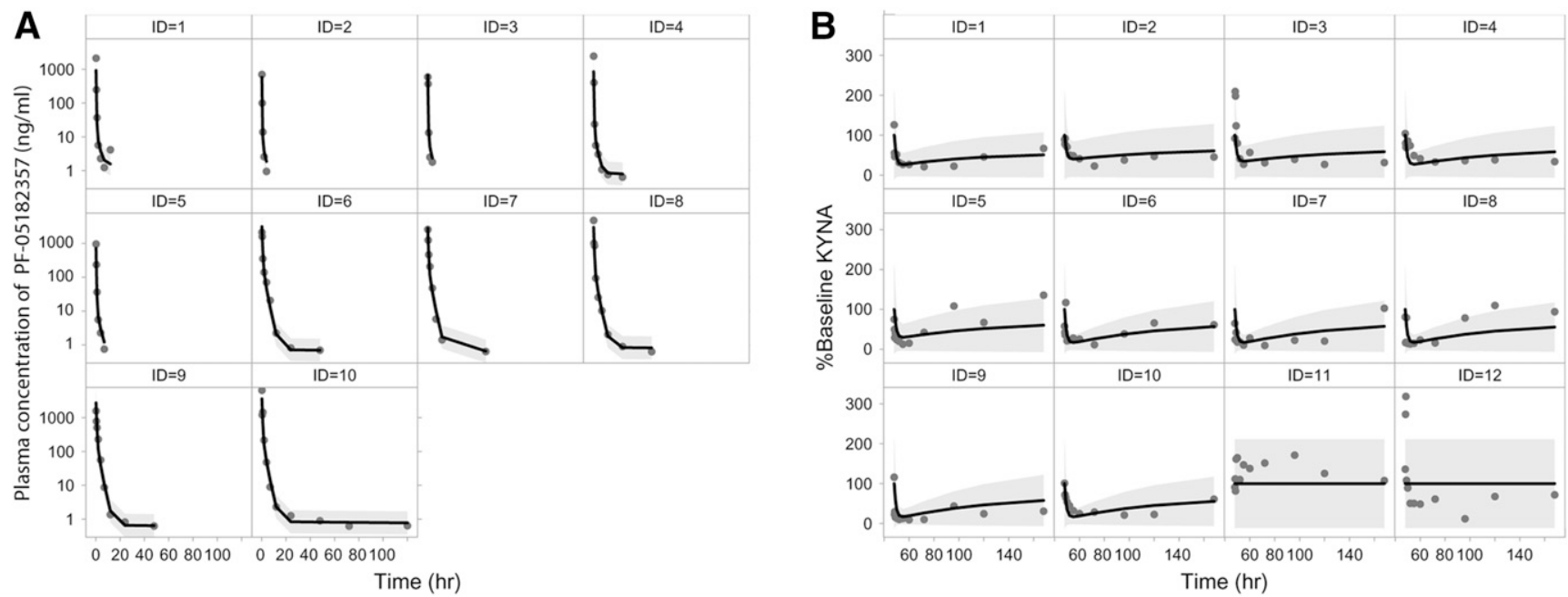

Fig. 6. Comparison of model-simulated time courses of PF-05182357 plasma concentrations (A) and CSF KYNA-lowering response (B) to corresponding observations for each individual NHP. Individuals (ID1-ID5) are from $1 \mathrm{mg} / \mathrm{kg}$ group; individuals (ID6-ID10) are from $3 \mathrm{mg} / \mathrm{kg}$ group; individuals (ID11 and ID 12) are from vehicle group. Solid circles represent observed data, solid lines represent the simulated median, and shaded area represents the 5th and 95th percentiles from model prediction. 


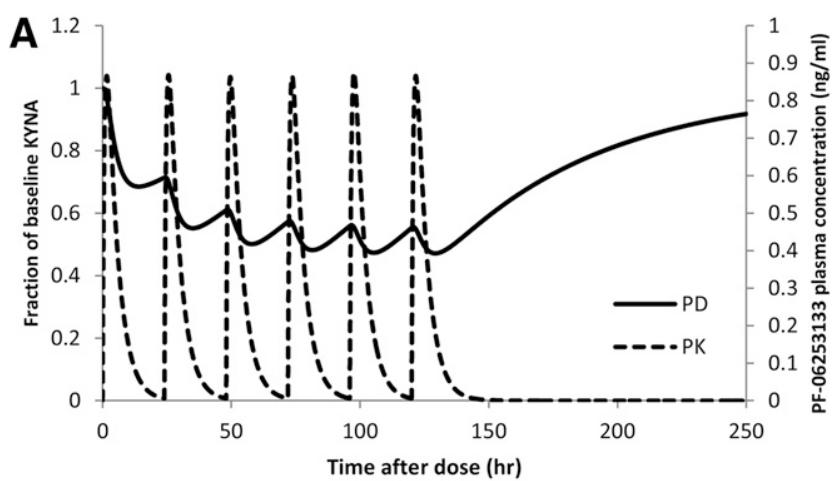

B

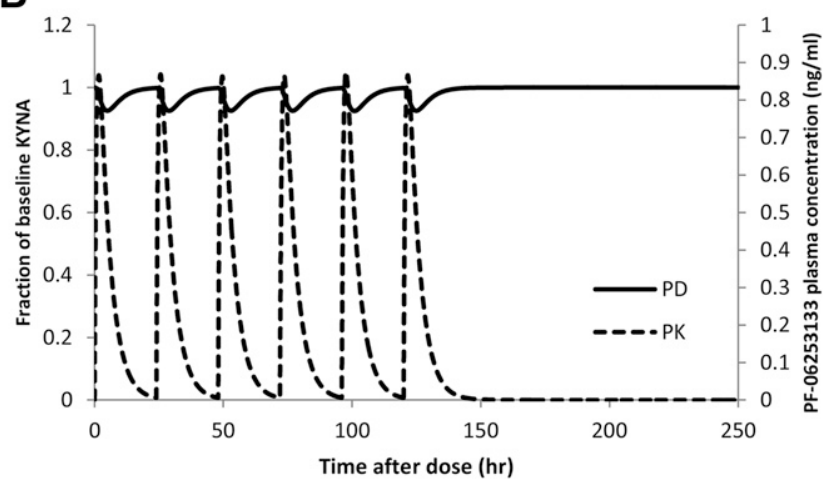

Fig. 7. Simulated clinical central KYNA-lowering effect after oral dosing of PF-06253133 based on the projected human KAT II turnover half-life of 43.4 hours (A) or the hypothetical KAT II turnover half-life of 1 hour (B). Solid line represents PD response (i.e., KYNA-lowering), and dashed line represents PK profile (i.e., PF-06253133 plasma concentration).

targets is less frequent. Our results illustrate that this is an important factor to consider for the development of smallmolecule irreversible inhibitors. Clearly, knowledge of KAT II turnover kinetics significantly accelerated the discovery of safe clinical candidates.

As illustrated in the preceding simulation, proper translational analysis is key to successful development of a clinical candidate based on preclinical information. One of the important factors in translational analysis is to understand compound potency differences between human and preclinical species (Chang et al., 2011). This could be particularly important for KAT II, as potency shifts between human and rodent KAT II have been previously reported for a reversible inhibitor, (S)-(4)-(ethylsulfonyl) benzoylalanine (Pellicciari et al., 2008). Since the target potency of different species is routinely evaluated in vitro, verifying the predictivity of in vitro assays in preclinical in vivo models becomes important. Model-estimated rat in vivo $k_{\text {inact }} / K_{\mathrm{I}}$ values sufficiently correlated with $k_{\text {inact }} / K_{I}$ estimates from primary rat mixed glia/astrocytes for PF-04859989, PF-05579960, and PF-06253133 (80.6 $\mathrm{M}^{-1} \mathrm{~s}^{-1}$ vs. $134 \mathrm{M}^{-1} \mathrm{~s}^{-1}, 535.4 \mathrm{M}^{-1} \mathrm{~s}^{-1} \mathrm{vs}$. $564 \mathrm{M}^{-1} \mathrm{~s}^{-1}$ and $848.6 \mathrm{M}^{-1} \mathrm{~s}^{-1}$ vs. $477 \mathrm{M}^{-1} \mathrm{~s}^{-1}$, respectively). When such potencies were compared across species for each compound, a significant shift was observed: they appear to be 10- to 50-fold more potent for human KAT II than for rat KAT II. Similar magnitude of potency shift ( $\sim 20$ fold) between humans and rats for another irreversible KAT II inhibitor was reported recently (Lu et al., 2016). Failure to consider this important factor in clinical study design could compromise the ability to fine-tune KYNA levels, potentially exposing the brain to significant KYNA deprivation, which could be deleterious to normal brain function in cases of overdosing in humans. Not surprisingly, our translational analyses suggest that the NHP model is more predictive of human brain KYNA-lowering profile via KAT II inhibition based on both similar compound potency $\left(16,668 \mathrm{M}^{-1} \mathrm{~s}^{-1} \mathrm{vs}\right.$. $13,895 \mathrm{M}^{-1} \mathrm{~s}^{-1}$ for PF-05182357 in NHPs and humans, respectively) and comparable KAT II turnover kinetics (KAT II half-life of 34.7 hours vs. 43.4 hours in NHPs and humans, respectively). This finding is further supported by KAT II enzyme sequence similarity across species, where humans shared more similarity with NHPs than with rats $(97 \%$ between humans and NPHs, $72 \%$ between humans and rats). Additionally, establishment of compound potency IVIVC suggests that prioritization of subsequent candidates can rely on the results from in vitro assays, as they are predictive of the outcome from more resource-intensive in vivo studies. In addition to reducing animal use, this approach also expedited the drug-discovery process (Gabrielsson et al., 2009).

In summary, mechanism-based PKPD modeling in translational research not only advanced the understanding of KAT II-KYNA biologic system, but it also facilitated efficient development of clinical candidates with high confidence of modulating brain KYNA levels via KAT II inhibition.

\section{Authorship Contributions}

Participated in research design: Chang, Fonseca, Zawadzke, Strick, Campbell, Dounay, Tuttle, Verhoest, Maurer.

Conducted experiments: Horner, Salafia, Welch, Gernhardt.

Contributed new reagents or analytic tools: Gernhardt, Dounay, Tuttle, Verhoest.

Performed data analysis: Chang, Fonseca, Li, Zawadzke, Rong, Sawant-Basak, Liras.

Wrote or contributed to the writing of the manuscript: Chang, Zawadzke, Verhoest, Maurer.

\section{References}

Abelö A, Eriksson UG, Karlsson MO, Larsson H, and Gabrielsson J (2000) A turnover model of irreversible inhibition of gastric acid secretion by omeprazole in the dog. $J$ Pharmacol Exp Ther 295:662-669.

Amori L, Guidetti P, Pellicciari R, Kajii Y, and Schwarcz R (2009) On the relationship between the two branches of the kynurenine pathway in the rat brain in vivo. $J$ Neurochem 109:316-325.

Chang C, Byon W, Lu Y, Jacobsen LK, Badura LL, Sawant-Basak A, Miller E, Liu J, Grimwood S, Wang EQ, et al. (2011) Quantitative PK-PD model-based translational pharmacology of a novel kappa opioid receptor antagonist between rats and humans. AAPS J 13:565-575.

Danhof M, de Jongh J, De Lange EC, Della Pasqua O, Ploeger BA, and Voskuyl RA (2007) Mechanism-based pharmacokinetic-pharmacodynamic modeling: biophase distribution, receptor theory, and dynamical systems analysis. Annu Rev Pharmacol Toxicol 47:357-400.

Dayneka NL, Garg V, and Jusko WJ (1993) Comparison of four basic models of indirect pharmacodynamic responses. J Pharmacokinet Biopharm 21:457-478.

Deigner HP, Kinscherf R, Claus R, Fyrnys B, Blencowe C, and Hermetter A (1999) Novel reversible, irreversible and fluorescent inhibitors of platelet-activating factor acetylhydrolase as mechanistic probes. Atherosclerosis 144:79-90.

DiNatale BC, Murray IA, Schroeder JC, Flaveny CA, Lahoti TS, Laurenzana EM, Omiecinski CJ, and Perdew GH (2010) Kynurenic acid is a potent endogenous aryl hydrocarbon receptor ligand that synergistically induces interleukin-6 in the presence of inflammatory signaling. Toxicol Sci 115(1):89-97.

Dounay AB, Anderson M, Bechle BM, Campbell BM, Claffey MM, Evdokimov A, Evrard E, Fonseca KR, Gan X, Ghosh S, et al. (2012) Discovery of brain-penetrant, irreversible kynurenine aminotransferase II inhibitors for schizophrenia. ACS Med Chem Lett 3:187-192.

Erhardt S, Blennow K, Nordin C, Skogh E, Lindström LH, and Engberg G (2001) Kynurenic acid levels are elevated in the cerebrospinal fluid of patients with schizophrenia. Neurosci Lett 313:96-98.

Ferron GM, McKeand W, and Mayer PR (2001) Pharmacodynamic modeling of pantoprazole's irreversible effect on gastric acid secretion in humans and rats. $J$ Clin Pharmacol 41:149-156.

Gabrielsson J, Dolgos H, Gillberg PG, Bredberg U, Benthem B, and Duker G (2009) Early integration of pharmacokinetic and dynamic reasoning is essential for optimal development of lead compounds: strategic considerations. Drug Discov Today 14:358-372. 
Guidetti P, Amori L, Sapko MT, Okuno E, and Schwarcz R (2007) Mitochondrial aspartate aminotransferase: a third kynurenate-producing enzyme in the mammalian brain. $J$ Neurochem 102:103-111.

Guidetti P, Okuno E, and Schwarcz R (1997) Characterization of rat brain kynurenine aminotransferases I and II. J Neurosci Res 50:457-465.

Han Q, Cai T, Tagle DA, and Li J (2010) Structure, expression, and function of kynurenine aminotransferases in human and rodent brains. Cell Mol Life Sci 67 : 353-368.

Henderson JL, Sawant-Basak A, Tuttle JB, Dounay AB, McAllister LA, Pandit J, Rong S, Hou X, Bechle BM, Kim J-Y, et al. (2013) Discovery of hydroxamate bioisosteres as KAT II inhibitors with improved oral bioavailability and pharmacokinetics. MedChemComm 4:125-129.

Hilmas C, Pereira EF, Alkondon M, Rassoulpour A, Schwarcz R, and Albuquerque EX (2001) The brain metabolite kynurenic acid inhibits alpha7 nicotinic receptor activity and increases non-alpha7 nicotinic receptor expression: physiopathological implications. $J$ Neurosci 21:7463-7473.

Homer A (1914) The constitution of kynurenic acid. $J$ Biol Chem 17:509-518.

Hutmacher MM, Mukherjee D, Kowalski KG, and Jordan DC (2005) Collapsing mechanistic models: an application to dose selection for proof of concept of a selective irreversible antagonist. $J$ Pharmacokinet Pharmacodyn 32:501-520.

Kalvass JC, Maurer TS, and Pollack GM (2007) Use of plasma and brain unbound fractions to assess the extent of brain distribution of 34 drugs: comparison of unbound concentration ratios to in vivo p-glycoprotein efflux ratios. Drug Metab Dispos 35:660-666.

Kessler M, Terramani T, Lynch G, and Baudry M (1989) A glycine site associated with N-methyl-D-aspartic acid receptors: characterization and identification of a new class of antagonists. J Neurochem 52:1319-1328.

Kiss C, Ceresoli-Borroni G, Guidetti P, Zielke CL, Zielke HR, and Schwarcz R (2003) Kynurenate production by cultured human astrocytes. J Neural Transm (Vienna) 110(1):1-14.

Koshy Cherian A, Gritton H, Johnson DE, Young D, Kozak R, and Sarter M (2014) A systemically-available kynurenine aminotransferase II (KAT II) inhibitor restores nicotine-evoked glutamatergic activity in the cortex of rats. Neuropharmacology 82:41-48.

Kozak R, Campbell BM, Strick CA, Horner W, Hoffmann WE, Kiss T, Chapin DS, McGinnis D, Abbott AL, Roberts BM, et al. (2014) Reduction of brain kynurenic acid improves cognitive function. J Neurosci 34:10592-10602.

Kuzmic P (1996) Program DYNAFIT for the analysis of enzyme kinetic data: application to HIV proteinase. Anal Biochem 237:260-273.

Kuzmic P (2009) DynaFit: a software package for enzymology. Methods Enzymol 467: $247-280$.

Linderholm KR, Alm MT, Larsson MK, Olsson SK, Goiny M, Hajos M, Erhardt S, and Engberg G (2016) Inhibition of kynurenine aminotransferase II reduces activity of midbrain dopamine neurons. Neuropharmacology 102:42-47.

Lu H, Kopcho L, Ghosh K, Witmer M, Parker M, Gupta S, Paul M, Krishnamurthy P, Laksmaiah B, Xie D, et al. (2016) Development of a RapidFire mass spectrometry assay and a fluorescence assay for the discovery of kynurenine aminotransferase II inhibitors to treat central nervous system disorders. Anal Biochem 501:56-65.

Luu KT, Bergqvist S, Chen E, Hu-Lowe D, and Kraynov E (2012) A model-based approach to predicting the human pharmacokinetics of a monoclonal antibody exhibiting target-mediated drug disposition. J Pharmacol Exp Ther 341:702-708.
Olsson SK, Samuelsson M, Saetre P, Lindström L, Jönsson EG, Nordin C, Engberg G, Erhardt S, and Landén M (2010) Elevated levels of kynurenic acid in the cerebrospinal fluid of patients with bipolar disorder. J Psychiatry Neurosci 35:195-199.

Passera E, Campanini B, Rossi F, Casazza V, Rizzi M, Pellicciari R, and Mozzarelli A (2011) Human kynurenine aminotransferase II: reactivity with substrates and inhibitors. FEBS J 278:1882-1900.

Paxinos G and Watson C (1982) The Rat Brain in Stereotaxic Coordinates, Academic Press, Sydney.

Pellicciari R, Venturoni F, Bellocchi D, Carotti A, Marinozzi M, Macchiarulo A, Amori L, and Schwarcz R (2008) Sequence variants in kynurenine aminotransferase II (KAT II) orthologs determine different potencies of the inhibitor S-ESBA. ChemMedChem 3:1199-1202.

Rempel BP and Withers SG (2008) Covalent inhibitors of glycosidases and their applications in biochemistry and biology. Glycobiology 18:570-586.

Robbins TW and Murphy ER (2006) Behavioural pharmacology: 40+ years of progress, with a focus on glutamate receptors and cognition. Trends Pharmacol Sci $\mathbf{2 7}$ 141-148.

Rossi F, Garavaglia S, Montalbano V, Walsh MA, and Rizzi M (2008) Crystal structure of human kynurenine aminotransferase II, a drug target for the treatment of schizophrenia. J Biol Chem 283:3559-3566.

Schmidt W, Guidetti P, Okuno E, and Schwarcz R (1993) Characterization of human brain kynurenine aminotransferases using $[3 \mathrm{H}]$ kynurenine as a substrate. $\mathrm{Neu}$ roscience 55:177-184

Schwarcz R, Rassoulpour A, Wu HQ, Medoff D, Tamminga CA, and Roberts RC (2001) Increased cortical kynurenate content in schizophrenia. Biol Psychiatry 50 521-530.

Schwartz PA, Kuzmic P, Solowiej J, Bergqvist S, Bolanos B, Almaden C, Nagata A Ryan K, Feng J, Dalvie D, et al. (2014) Covalent EGFR inhibitor analysis reveals importance of reversible interactions to potency and mechanisms of drug resistance. Proc Natl Acad Sci USA 111:173-178.

Singh K, Orr JM, and Abbott FS (1988) Pharmacokinetics and enterohepatic circulation of 2-n-propyl-4-pentenoic acid in the rat. Drug Metab Dispos 16:848-852.

Thomsen MS, Hansen HH, Timmerman DB, and Mikkelsen JD (2010) Cognitive improvement by activation of alpha7 nicotinic acetylcholine receptors: from animal models to human pathophysiology. Curr Pharm Des 16:323-343.

Tuttle JB, Anderson M, Bechle BM, Campbell BM, Chang C, Dounay AB, Evrard E, Fonseca KR, Gan X, Ghosh S, et al. (2012) Structure-based design of irreversible human KAT II inhibitors: discovery of new potency-enhancing interactions. ACS Med Chem Lett 4:37-40.

Zhang L, Beal SL, and Sheiner LB (2003) Simultaneous vs. sequential analysis for population PK/PD data I: best-case performance. J Pharmacokinet Pharmacodyn 30:387-404.

Zhao L, Shang EY, and Sahajwalla CG (2012) Application of pharmacokineticspharmacodynamics/clinical response modeling and simulation for biologics drug development. J Pharm Sci 101:4367-4382.

Address correspondence to: Dr. Cheng Chang, Clinical Pharmacology, Pfizer Global Product Development, MS 8260-2429, Pfizer Inc., Eastern Point Road, Groton, CT 06340. E-mail: cheng.chang@pfizer.com 